\section{An Inconvenient Truth Concerning Surgery for Mesothelioma}

To THE EDITOR: A recently published ASCO clinical practice guideline discussed the treatment of malignant pleural mesothelioma. ${ }^{1}$ The authors are commended for their efforts in producing a guideline in this important area. The ASCO guideline methodology states that to return a "strong" recommendation, there should be high confidence that the recommendation reflects best practice. This should be based on: (1) strong evidence for a true net effect (eg, benefits exceed harm); (2) consistent results, with no or minor exceptions; (3) minor or no concerns about study quality; and/or (4) the extent of the Expert Panelists' agreement.

On the basis of these criteria, we do not believe that the following "strong" recommendations in the guideline regarding surgical cytoreduction with extrapleural pneumonectomy (EPP) or pleurectomy/decortication $(\mathrm{P} / \mathrm{D})$ reflect the published literature: "In selected patients with early-stage disease, it is strongly recommended that a maximal surgical cytoreduction should be performed"1 and "maximal surgical cytoreduction involves either EPP or lung-sparing options (P/D, extended P/D)."

The authors assert that this recommendation is supported by the published literature. The evidence quoted, however, is limited to case series $^{2,3}$ from which, as retrospective, nonrandomized data, only limited conclusions can be drawn. In one referenced series, Bovolato et $\mathrm{al}^{3}$ reported that a modest benefit was observed with surgery combined with systemic therapy. ${ }^{3}$ This conclusion was based, however, on a retrospective comparison of overall survival (OS) between patients treated medically, with chemotherapy or best supportive care, and those treated surgically with $\mathrm{P} / \mathrm{D}$ or EPP. ${ }^{3}$ Unsurprisingly, there was significant heterogeneity between the groups in this study. The researchers acknowledged that when their analyses were restricted to patients with good prognostic factors (as would usually be the case for surgical patients) there was no statistically significant difference in OS between study groups. The review by Sharif et al, ${ }^{2}$ quoted in the guideline, concluded that EPP provides no benefit for symptom control or OS compared with supportive care. The Mesothelioma and Radical Surgery (MARS) feasibility trial remains the only randomized study conducted in this field. ${ }^{4}$ It is surprising that it is not referenced in the relevant section of the guideline. MARS attempted a head-to-head comparison of surgery (EPP) versus no surgery but was terminated after the 50-patient feasibility phase because of the high morbidity associated with EPP and better survival in the nonsurgical arm. ${ }^{4}$ Survival was significantly better in the comparator arm, even though three patients in the comparator arm elected to have EPP off trial at another center and were therefore exposed to the risk of harm from EPP. It is mystifying how the guideline group can conclude that there is strong evidence to recommend surgery with EPP for this patient group.

Similarly, there are no randomized controlled trials demonstrating a benefit for $\mathrm{P} / \mathrm{D}$ or extended $\mathrm{P} / \mathrm{D}$ for mesothelioma. Given the significant morbidity associated with radical mesothelioma surgery and the absence of any proven benefit, in our opinion, it would have been more appropriate for the guideline authors to conclude that if surgery in any form were to be contemplated for a patient with mesothelioma, it should take place in the context of a properly conducted randomized controlled trial.

The patent lack of high-quality surgical evidence has, in fact, resulted in the funding of a large randomized controlled trial in the United Kingdom. MARS 2 (ClinicalTrials.gov identifier: NCT02040272) randomly assigns patients to EPD or no surgery, in combination with pemetrexed/cisplatin chemotherapy, to address the currently unanswered question regarding the role of EPD in malignant pleural mesothelioma management. It is currently recruiting well, and we await these important results with interest.

Finally, we would also like to draw the authors' attention to their recommendation that the optimal management of symptomatic pleural effusions in mesothelioma includes video-assisted thoracoscopic surgery (VATS) decortication and pleurodesis. This is one area where there is strong randomized controlled data comparing VATSpartial pleurectomy (VATS-PP) with talc pleurodesis. The MesoVATS trial, published in The Lancet Oncology in 2014, demonstrated that VATS-PP offered no survival benefit at 12 months over talc poudrage/slurry and was associated with more complications and a longer hospital stay. ${ }^{5}$ Despite acknowledging this information in their literature review, the authors recommend that VATS-PP be offered as an option for management of pleural effusion.

Surgery for mesothelioma, whether with radical or palliative intent, has been shown in well-conducted randomized controlled trials to confer no benefit in overall survival or quality of life. Although this truth may be inconvenient for providers of such surgery, it deserves greater recognition in the guideline recommendations of an organization with the worldwide reputation and influence of ASCO.

\section{Duneesha de Fonseka}

University of Bristol, North Bristol NHS Trust, Bristol, United Kingdom

\section{Mark Slade}

Gloucestershire Hospitals NHS Foundation Trust, Cheltenham, United Kingdom

Kevin G. Blyth

Queen Elizabeth University Hospital and University of Glasgow, United Kingdom

\section{John Edwards}

Sheffield Teaching Hospitals, Sheffield, United Kingdom

\section{Matthew Evison}

Wythenshawe Hospital, Manchester, UK

\section{Mark Roberts}

Sherwood Forest Hospitals NHS Foundation Trust, Sutton in Ashfield, United Kingdom

\section{Najib Rahman}

University of Oxford, Oxford, United Kingdom

\section{Ian Woolhouse}

University Hospitals of Birmingham, Birmingham, United Kingdom

\section{Nick A. Maskell}

University of Bristol, North Bristol NHS Trust, Bristol, United Kingdom 
AUTHORS' DISCLOSURES OF POTENTIAL CONFLICTS OF INTEREST

Disclosures provided by the authors are available with this article at jco.org.

\section{REFERENCES}

1. Kindler HL, Ismaila N, Armato SG III, et al: Treatment of malignant pleural mesothelioma: American Society of Clinical Oncology clinical practice guideline. J Clin Oncol 36:1343-1373, 2018

2. Sharif $S$, Zahid I, Routledge T, et al: Extrapleural pneumonectomy or supportive care: Treatment of malignant pleural mesothelioma? Interact Cardiovasc Thorac Surg 12:1040-1045, 2011

3. Bovolato $P$, Casadio $C$, Billè $A$, et al: Does surgery improve survival of patients with malignant pleural mesothelioma?: A multicenter retrospective analysis of 1365 consecutive patients. J Thorac Oncol 9 : 390-396, 2014

4. Treasure T, Lang-Lazdunski L, Waller D, et al: Extra-pleural pneumonectomy versus no extra-pleural pneumonectomy for patients with malignant pleural mesothelioma: Clinical outcomes of the Mesothelioma and Radical Surgery (MARS) randomised feasibility study. Lancet Oncol 12:763-772, 2011

5. Rintoul RC, Ritchie AJ, Edwards JG, et al: Efficacy and cost of videoassisted thoracoscopic partial pleurectomy versus talc pleurodesis in patients with malignant pleural mesothelioma (MesoVATS): An open-label, randomised, controlled trial. Lancet 384:1118-1127, 2014

DOI: https://doi.org/10.1200/JCO.2018.78.6590; published at jco.org on June 14, 2018. 


\section{AUTHORS' DISCLOSURES OF POTENTIAL CONFLICTS OF INTEREST}

An Inconvenient Truth Concerning Surgery for Mesothelioma

The following represents disclosure information provided by authors of this manuscript. All relationships are considered compensated. Relationships are self-held unless noted. I = Immediate Family Member, Inst = My Institution. Relationships may not relate to the subject matter of this manuscript. For more information about ASCO's conflict of interest policy, please refer to www.asco.org/rwc or ascopubs.org/jco/site/ifc.

\section{Duneesha de Fonseka}

No relationship to disclose

Mark Slade

No relationship to disclose

Kevin G. Blyth

Research Funding: Rocket Medical UK, SomaLogic

\section{John Edwards}

No relationship to disclose

\section{Matthew Evison}

No relationship to disclose

\section{Mark Roberts}

No relationship to disclose

\section{Najib Rahman}

Consulting or Advisory Role: Rocket Medical UK

Ian Woolhouse

No relationship to disclose

Nick A. Maskell

Honoraria: $\mathrm{BD}$

Consulting or Advisory Role: Becton Dickinson

Research Funding: Becton Dickinson (Inst) 